\title{
Existence and uniqueness of positive solutions for a class of nonlinear fractional differential equations
}

\author{
Lingling Zhang ${ }^{1 *}$ and Huimin Tian ${ }^{1,2}$
}

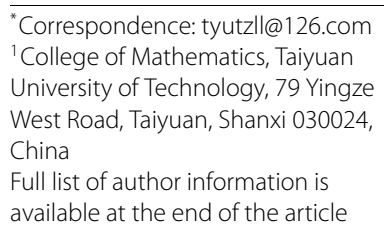

available at the end of the article

\begin{abstract}
In the article, the existence and uniqueness of positive solutions for a class of fractional differential equation with nonlinear boundary conditions are discussed. By applying some fixed point theorems on cone, we gain a unique positive solution and construct two iterative sequences to approximate the solution. Moreover, as applications of our main results, some examples are also presented.
\end{abstract}

Keywords: fractional differential equations; fixed point theorem; mixed monotone operator; positive solution

\section{Introduction}

As is well known, fractional differential equations has been widely applied to various fields of physical, engineering, biology, chemistry, etc.; see [1-6]. Recently, more and more authors have paid attention to the research on the solutions for fractional differential equations. There are many great results as regards positive solutions of boundary value problems (see [7-19]). For example, Zhai, Yan and Yang [7] studied the existence and uniqueness of positive solutions to fractional differential equation boundary value problems. In [10], Yuan was concerned with the $(n-1,1)$-type semipositone conjugate boundary value problems. Yang [14] and the authors [17] considered nonlinear fractional differential equations with integral boundary conditions. In [15], the authors were interested in the nonlinear fractional differential equation with a derivative term.

In this paper, we study the nonlinear fractional differential equation given by

$$
\left\{\begin{array}{l}
D_{0^{+}}^{\nu} x(t)+f\left(t, x(t), D_{0^{+}}^{\gamma} x(t)\right)+g(t, x(t))=0, \quad t \in(0,1), n-1<v<n \\
x^{(i)}(0)=0, \quad i=0,1,2,3, \ldots, n-2 ; \\
{\left[D_{0^{+}}^{\beta} x(t)\right]_{t=1}=k(x(1))}
\end{array}\right.
$$

where $n>3,1 \leq \gamma \leq \beta \leq n-2, f:[0,1] \times[0, \infty) \times[0, \infty) \rightarrow[0, \infty), g:[0,1] \times[0, \infty) \rightarrow$ $[0, \infty)$, and $k:[0, \infty) \rightarrow[0, \infty)$ are continuous functions, $D_{0^{+}}^{v}$ stands for the RiemannLiouville fractional derivative of order $v$, and $x^{(i)}$ represents the $i$ th (ordinary) derivative of $x$. 
When $k(x)=0$ and $f\left(t, x, D_{0^{+}}^{\gamma} x(t)\right)=0$ in (1.1), the problem (1.1) is changed into the form of the following fractional differential equation considered in [8]:

$$
\left\{\begin{array}{l}
D_{0^{+}}^{v} x(t)+g(t, x(t))=0, \quad t \in(0,1), n-1<v \leq n \\
x^{(i)}(0)=0, \quad i=0,1,2,3, \ldots, n-2 \\
{\left[D_{0^{+}}^{\beta} x(t)\right]_{t=1}=0, \quad 1 \leq \beta \leq n-2}
\end{array}\right.
$$

By means of the Krasnosel'skii fixed point theorem in cones, Goodrich only came to the conclusion that problem (1.2) has at least one positive solution. Meanwhile, he did not mention the uniqueness.

When $k(x)=0$ and $\gamma=0$ in (1.1), Mohamed Jleli and Bessem Samet [9] considered the following problem:

$$
\left\{\begin{array}{l}
D_{0^{+}}^{v} x(t)+f(t, x(t), x(t))+g(t, x(t))=0, \quad t \in(0,1), n-1<v \leq n \\
x^{(i)}(0)=0, \quad i=0,1,2,3, \ldots, n-2 \\
{\left[D_{0^{+}}^{\beta} x(t)\right]_{t=1}=0, \quad 2 \leq \beta \leq n-2 .}
\end{array}\right.
$$

They investigated a unique positive solution of problem (1.3) by using a fixed point theorem of a mixed monotone operator.

Compared with [8] and [9], we not only generalize the boundary conditions but also consider the derivative term. As far as we know, few papers can be found in the literature on the existence and uniqueness of positive solutions for the (1.1), especially the analysis relying on fixed point theorems of a sum operator. Our main contribution in this paper is to give some distinct methods to obtain the main results of $[8,9]$. By some fixed theorems of a sum operator on cone, we establish a unique solution and set up two iterative schemes to approximate the solution.

The construction of this paper is displayed as follows. In Section 2, we recall some basic definitions and notations and several useful lemmas. Section 3 contains the existence and uniqueness of solution for nonlinear fractional differential equations (1.1), based on some fixed point theorems. In Section 4, we give some examples to illustrate our main results.

\section{Preliminaries}

For convenience, we present some basic definitions, lemmas and preliminary results that will be used in this manuscript.

Definition 2.1 ([3]) The Riemann-Liouville fractional derivative of order $(p>0)$ of a function $h \in C[0,1]$ is given by

$$
D_{0^{+}}^{p} h(t)=\frac{1}{\Gamma(n-p)}\left(\frac{\mathrm{d}}{\mathrm{d} t}\right)^{n} \int_{0}^{t} \frac{h(s)}{(t-s)^{p-n+1}} \mathrm{~d} s .
$$

The Riemann-Liouville fractional integral of order $(q>0)$ of $h$ is given by

$$
I_{0^{+}}^{p} h(t)=\frac{1}{\Gamma(p)} \int_{0}^{t}(t-s)^{p-1} h(s) \mathrm{d} s
$$

where $n=[p]+1,[p]$ denotes the integer part of number $p$, provided that the right side is pointwise defined on $(0,1)$. 
Lemma 2.1 ([3]) Assume $h \in C[0,1], q \geq p \geq 0$, then

$$
D_{0^{+}}^{p} I_{0^{+}}^{q} h(t)=I_{0^{+}}^{q-p} h(t) .
$$

Lemma $2.2([3])$ Let $h \in C[0,1] \cap L^{1}[0,1], p>0$, then

$$
I_{0^{+}}^{p} D_{0^{+}}^{p} h(t)=c_{1} t^{p-1}+c_{2} t^{p-2}+\cdots+c_{n} t^{p-n}
$$

where $c_{i} \in R, i=1,2,3, \ldots, n(n=[p]+1)$.

Lemma 2.3 Let $h \in C[0,1]$, then the unique solution of the linear problem

$$
\begin{aligned}
& D_{0^{+}}^{v} x(t)+h(t)=0, \quad t \in(0,1), n-1<v \leq n ; \\
& x^{i}(0)=0, \quad i=0,1,2,3, \ldots, n-2 ; \\
& {\left[D_{0^{+}}^{\beta} x(t)\right]_{t=1}=k(x(1)), \quad 1 \leq \beta \leq n-2,}
\end{aligned}
$$

is given by

$$
x(t)=\int_{0}^{1} G(t, s) h(s) \mathrm{d} s+\frac{\Gamma(\nu-\beta)}{\Gamma(v)} k(x(1)) t^{\nu-1},
$$

where

$$
G(t, s)= \begin{cases}\frac{t^{\nu-1}(1-s)^{\nu-\beta-1}-(t-s)^{\nu-1}}{\Gamma(\nu)}, & 0 \leq s \leq t \leq 1 ; \\ \frac{t^{\nu-1}(1-s)^{\nu-\beta-1}}{\Gamma(\nu)}, & 0 \leq t \leq s \leq 1,\end{cases}
$$

is the Green's function.

Proof In view of Lemma 2.2 and (2.1), we have

$$
x(t)=-I_{0^{+}}^{\nu} h(t)+c_{1} t^{\nu-1}+c_{2} t^{\nu-2}+\cdots+c_{n} t^{\nu-n} .
$$

The boundary condition (2.2) implies that $c_{n}=c_{n-1}=c_{n-2}=\cdots=c_{2}=0$. Thus, the solution of (2.1) is equivalent to the following one:

$$
x(t)=-I_{0^{+}}^{v} h(t)+c_{1} t^{\nu-1} .
$$

Using the equality $D_{0^{+}}^{\alpha} t^{\beta}=\frac{\Gamma(\beta+1)}{\Gamma(\beta-\alpha+1)} t^{\beta-\alpha}$ and Lemma 2.1, (2.5) reduces to

$$
\begin{aligned}
D_{0^{+}}^{\beta} x(t) & =-I_{0^{+}}^{\nu-\beta} h(t)+\frac{\Gamma(v)}{\Gamma(v-\beta)} t^{\nu-\beta-1} c_{1} \\
& =-\int_{0}^{t} \frac{(t-s)^{\nu-\beta-1}}{\Gamma(\nu-\beta)} h(s) \mathrm{d} s+\frac{\Gamma(\nu)}{\Gamma(\nu-\beta)} t^{\nu-\beta-1} c_{1} .
\end{aligned}
$$

From (2.3) and (2.6), then we conclude

$$
-\int_{0}^{1} \frac{(1-s)^{\nu-\beta-1}}{\Gamma(v-\beta)} h(s) \mathrm{d} s+\frac{\Gamma(v)}{\Gamma(\nu-\beta)} c_{1}=k(x(1)) .
$$


Equation (2.7) may be simplified to get

$$
c_{1}=\frac{\Gamma(v-\beta)}{\Gamma(v)} k(x(1))+\int_{0}^{1} \frac{(1-s)^{v-\beta-1}}{\Gamma(v)} h(s) \mathrm{d} s .
$$

Finally, plugging (2.8) into (2.5), the unique solution of problem (2.1), (2.2), (2.3) is given by

$$
\begin{aligned}
x(t) & =-I_{0^{+}}^{v} h(t)+t^{\nu-1}\left\{\frac{\Gamma(\nu-\beta)}{\Gamma(\nu)} k(x(1))+\int_{0}^{1} \frac{(1-s)^{\nu-\beta-1}}{\Gamma(\nu)} h(s) \mathrm{d} s\right\} \\
& =-\int_{0}^{t} \frac{(t-s)^{\nu-1}}{\Gamma(\nu)} h(s) \mathrm{d} s+\frac{\Gamma(v-\beta)}{\Gamma(\nu)} k(x(1)) t^{\nu-1}+\int_{0}^{1} \frac{(1-s)^{\nu-\beta-1} t^{\nu-1}}{\Gamma(\nu)} h(s) \mathrm{d} s \\
& =\int_{0}^{1} G(t, s) h(s) \mathrm{d} s+\frac{\Gamma(v-\beta)}{\Gamma(\nu)} k(x(1)) t^{\nu-1},
\end{aligned}
$$

where $G(t, s)$ defined as (2.4).

Lemma 2.4 The Green's function (2.4) has the following properties:

$$
\begin{aligned}
0 & \leq t^{\nu-1}(1-s)^{\nu-\beta-1}\left[1-(1-s)^{\beta}\right] \leq \Gamma(\nu) G(t, s) \leq t^{\nu-1}(1-s)^{\nu-\beta-1}, \quad t, s \in[0,1], \\
0 & \leq t^{\nu-\gamma-1}(1-s)^{\nu-\beta-1}\left[1-(1-s)^{\beta-\gamma}\right] \leq \Gamma(\nu-\gamma) D_{0^{+}}^{\gamma} G(t, s) \\
& \leq t^{\nu-\gamma-1}(1-s)^{\nu-\beta-1}, \quad t, s \in[0,1] .
\end{aligned}
$$

Proof The first inequality (2.9) has been proved by [9]. Now we prove the inequality (2.10). From (2.4), we get

$$
D_{0^{+}}^{\gamma} G(t, s)= \begin{cases}\frac{t^{\nu-\gamma-1}(1-s)^{\nu-\beta-1}-(t-s)^{\nu-\gamma-1}}{\Gamma(\nu-\gamma)}, & 0 \leq s \leq t \leq 1 \\ \frac{t^{\nu-\gamma-1}(1-s)^{\nu-\beta-1}}{\Gamma(\nu-\gamma)}, & 0 \leq t \leq s \leq 1\end{cases}
$$

We can find that the right term in (2.10) is clear. So we only prove the right term. If $0 \leq$ $t \leq s \leq 1$, since $\beta \geq \gamma$, then

$$
\begin{aligned}
\Gamma(\nu-\gamma) G(t, s) & =t^{\nu-\gamma-1}(1-s)^{\nu-\beta-1} \\
& \geq t^{\nu-\gamma-1}(1-s)^{\nu-\beta-1}\left[1-(1-s)^{\beta-\gamma}\right] .
\end{aligned}
$$

If $0 \leq s \leq t \leq 1$, since $n-1<v \leq n$ with $n>3$ and $1 \leq \gamma \leq n-2$, then we get $t-s \leq t-t s=$ $(1-s) t$, which implies that

$$
(t-s)^{\nu-\gamma-1} \leq(1-s)^{\nu-\gamma-1} t^{\nu-\gamma-1} .
$$

Then

$$
\begin{aligned}
\Gamma(\nu-\gamma) G(t, s) & =t^{\nu-\gamma-1}(1-s)^{\nu-\beta-1}-(t-s)^{\nu-\gamma-1} \\
& \geq t^{\nu-\gamma-1}(1-s)^{\nu-\beta-1}-(1-s)^{\nu-\gamma-1} t^{\nu-\gamma-1} \\
& =t^{\nu-\gamma-1}(1-s)^{\nu-\beta-1}\left[1-(1-s)^{\beta-\gamma}\right] .
\end{aligned}
$$

Therefore equation (2.10) is demonstrated. 
In the sequel, we state here some basic concepts in ordered Banach spaces and some fixed point theorems, which will be used later.

Suppose that $(E,\|\cdot\|)$ is a real Banach space, which is partially ordered by a cone $P \subset E$, i.e., $x \leq y$ if and only if $y-x \in P$. If $x \leq y$ and $x \neq y$, then we denote $x<y$ or $y>x$. By $\theta$ we denote the zero element of $E$. Recall that a nonempty closed convex set $P \subset E$ is a cone if it satisfies: (1) $x \in P, r>0 \Rightarrow r x \in P$; (2) $x \in P,-x \in P \Rightarrow x=\theta$.

$P$ is called normal if $\exists N>0$ such that $\theta \leq x \leq y$ implies $\|x\| \leq N\|y\|$. In this case, the smallest constant satisfying this inequality is called the normality constant of $P$. For all $x, y \in E$, the notation $x \sim y$ means that there exist $\lambda>0, \mu>0$ such that $\lambda y \leq x \leq \mu y$. Clearly, $\sim$ is an equivalence relation. Given $h>\theta$, we denote by $P_{h}$ the set $P_{h}=\{x \in E \mid x \sim$ $h$. It is easy to see that $P_{h} \subset P$.

Definition 2.2 An operator $A: P \rightarrow P$ is said to be sub-homogeneous if it satisfies

$$
A(t x) \geq t A x, \quad \forall t \in(0,1), x \in P .
$$

Definition 2.3 Let $D=P$ or $D=\stackrel{\circ}{P}$ and $\alpha$ be a real number with $0 \leq \alpha<1$. An operator $A: D \rightarrow D$ is said to be $\alpha$-concave if it satisfies

$$
A(t x) \geq t^{\alpha} A x, \quad \forall t \in(0,1), x \in D .
$$

Definition 2.4 ([8]) An operator $A: P \times P \rightarrow P$ is said to be a mixed monotone operator if $A(x, y)$ is increasing in first component and decreasing in second component, i.e., $u_{i}, v_{i}(i=$ $1,2) \in P, u_{1} \leq u_{2}, v_{1} \geq v_{2}$, implies $A\left(u_{1}, v_{1}\right) \leq A\left(u_{2}, v_{2}\right)$. An element $x \in P$ is called a fixed point of $A$ if $A(x, x)=x$.

In [20], the operator equation $A x+B x+C(x, x)=x$ is considered. Here, the operator $A: P \rightarrow P$ is an increasing, $B: P \rightarrow P$ is a decreasing operator, $C: P \times P \rightarrow P$ is a mixed monotone operator. Then Wang and Zhang get the following main results.

Lemma 2.5 Let $\alpha \in(0,1)$ and $P$ is a normal cone of E. Assume that $A$ is sub-homogeneous and $B, C$ satisfy the following conditions:

$$
B\left(t^{-1} y\right) \geq t B(y), \quad C\left(t x, t^{-1} y\right) \geq t^{\alpha} C(x, y), \quad \forall t \in(0,1), x, y \in P .
$$

\section{Suppose that}

$\left(A_{1}\right)$ there exists $h_{0} \in P_{h}$ such that $A\left(h_{0}\right) \in P_{h}, B\left(h_{0}\right) \in P_{h}, C\left(h_{0}, h_{0}\right) \in P_{h}$;

$\left(A_{2}\right)$ there exists a constant $\delta>0$ such that $C(x, y) \geq \delta(A x+B y), \forall x, y \in P$.

\section{Then}

(1) $A: P_{h} \rightarrow P_{h}, B: P_{h} \rightarrow P_{h}, C: P_{h} \times P_{h} \rightarrow P_{h}$;

(2) there exist $u_{0}, v_{0} \in P_{h}$ and $r \in(0,1)$ such that

$$
r v_{0} \leq u_{0}<v_{0}, \quad u_{0} \leq A u_{0}+B v_{0}+C\left(u_{0}, v_{0}\right) \leq A v_{0}+B u_{0}+C\left(v_{0}, u_{0}\right) \leq v_{0}
$$

(3) the operator equation $A x+B x+C(x, x)=x$ has a unique solution $x^{*}$ in $P_{h}$; 
(4) for any initial values $x_{0}, y_{0} \in P_{h}$, constructing successively the sequences

$$
\begin{aligned}
& \qquad x_{n}=A x_{n-1}+B y_{n-1}+C\left(x_{n-1}, y_{n-1}\right), \quad n=1,2, \ldots, \\
& y_{n}=A y_{n-1}+B x_{n-1}+C\left(y_{n-1}, x_{n-1}\right), \quad n=1,2, \ldots, \\
& \text { we have } x_{n} \rightarrow x^{*} \text { and } y_{n} \rightarrow x^{*} \text { as } n \rightarrow \infty
\end{aligned}
$$

Lemma 2.6 Let $\alpha \in(0,1)$ and $P$ is a normal cone of $E$. Assume that A sub-homogeneous operator, and the following inequalities hold for $B, C$ :

$$
B\left(t^{-1} y\right) \geq t^{\alpha} B(y), \quad C\left(t x, t^{-1} y\right) \geq t C(x, y), \quad \forall t \in(0,1), x, y \in P .
$$

Suppose that

$\left(A_{1}^{\prime}\right)$ there exists $h_{0} \in P_{h}$ such that $A\left(h_{0}\right) \in P_{h}, B\left(h_{0}\right) \in P_{h}, C\left(h_{0}, h_{0}\right) \in P_{h}$;

$\left(A_{2}^{\prime}\right)$ there exists a constant $\delta>0$ such that $A x+C(x, y) \leq \delta B y, \forall x, y \in P$.

Then the conclusions (1)-(4) in Lemma 2.5 hold.

Lemma 2.7 Let $\alpha \in(0,1)$ and $P$ is a normal cone of $E$. Assume that $A$ is a $\alpha$-concave operator, the operators $B, C$ satisfy

$$
B\left(t^{-1} y\right) \geq t B(y), \quad C\left(t x, t^{-1} y\right) \geq t C(x, y), \quad \forall t \in(0,1), x, y \in P .
$$

\section{Suppose that}

$\left(A_{1}^{\prime \prime}\right)$ there exists $h_{0} \in P_{h}$ such that $A\left(h_{0}\right) \in P_{h}, B\left(h_{0}\right) \in P_{h}, C\left(h_{0}, h_{0}\right) \in P_{h}$;

$\left(A_{2}^{\prime \prime}\right)$ there exists a constant $\delta>0$ such that $B y+C(x, y) \leq \delta A x, \forall x, y \in P$.

Then the conclusions (1)-(4) in Lemma 2.5 hold.

\section{Main results}

In the section, we use Lemmas 2.5, 2.6, 2.7 to obtain the existence and uniqueness of positive solutions for the problem (1.1).

Set $E=\left\{x \mid x \in C[0,1], D_{0^{+}}^{\gamma} x \in C[0,1]\right\}$ is a Banach space with the norm

$$
\|x(t)\|=\max \left\{\max _{t \in[0,1]}|x(t)|, \max _{t \in[0,1]} D_{0^{+}}^{\gamma}|x(t)|\right\} .
$$

$E$ is endowed with an order relation $u \preceq v$ if $u(t) \leq v(t), D_{0^{+}}^{\gamma} u(t) \leq D_{0^{+}}^{\gamma} v(t)$. Moreover, let $P \subset E$ be defined by

$$
P=\left\{x \in E: x(t) \geq 0, D_{0^{+}}^{\gamma} x(t) \geq 0, \forall t \in[0,1]\right\} .
$$

Clearly, $P$ is a normal cone and $P_{h} \subset E$.

By Lemma 2.3, we know that the unique solution for the problem (1.1) has an integral formulation:

$$
x(t)=\int_{0}^{1} G(t, s) f\left(s, x(s), D_{0^{+}}^{\gamma} x(s)\right) \mathrm{d} s+\int_{0}^{1} G(t, s) g(s, x(s)) \mathrm{d} s+\frac{\Gamma(v-\beta)}{\Gamma(v)} k(x(1)) t^{\nu-1},
$$

where $G(t, s)$ is given by $(2.4)$. 
We define the operators $A, B, C$ :

$$
\begin{aligned}
& A(u)(t)=\int_{0}^{1} G(t, s) g(s, u(s)) \mathrm{d} s, \quad t \in[0,1] \\
& B(v)(t)=\frac{\Gamma(v-\beta)}{\Gamma(v)} k(v(1)) t^{\nu-1}, \quad t \in[0,1] ; \\
& C(u, v)(t)=\int_{0}^{1} G(t, s) f\left(s, u(s), D_{0^{+}}^{\gamma} v(s)\right) \mathrm{d} s, \quad t \in[0,1] .
\end{aligned}
$$

Evidently $u$ is the solution of problem (1.1) if and only if $u=A(u)+B(u)+C(u, u)$.

\section{Theorem 3.1 Assume that}

$\left(H_{1}\right) f:[0,1] \times[0,+\infty) \times[0,+\infty) \rightarrow[0,+\infty), g:[0,1] \times[0,+\infty) \rightarrow[0,+\infty)$, and $k:$ $[0,+\infty) \rightarrow[0,+\infty)$ are continuous;

$\left(H_{2}\right) f(t, x, y)$ is increasing in $x \in[0,+\infty)$ for fixed $t \in[0,1]$ and $y \in[0,+\infty)$, decreasing in $y \in[0,+\infty)$ for fixed $t \in[0,1]$ and $x \in[0,+\infty), g(t, x)$ is increasing in $x \in[0,+\infty)$ for fixed $t \in[0,1], k(y)$ is decreasing in $y \in[0,+\infty)$ for fixed $t \in[0,1]$ with $k(y(1)) \neq 0$;

$\left(H_{3}\right)$ there exists a constant $\alpha \in(0,1)$ such that $f\left(t, \eta x, \eta^{-1} y\right) \geq t^{\alpha} f(t, x, y), \forall \eta \in(0,1), t \in$ $[0,1], x, y \in[0,+\infty) ; g(t, x), k(y)$ satisfy $g(t, \eta x) \geq \eta g(t, x), k\left(\eta^{-1} y\right) \geq \eta k(y), \forall \eta \in(0,1)$, $x, y \in[0,+\infty)$;

$\left(H_{4}\right) g(t, 0) \not \equiv 0$ for $t \in[0,1]$ and there exist two constants $\delta_{1}>0$ and $\delta_{2}>0$ such that $f(t, x, y) \geq \delta_{1} g(t, x), f(t, x, y) \geq \delta_{2} \geq k(y), t \in[0,1], x, y \in[0,+\infty)$.

Then we have the following conclusions:

(1) there exist $u_{0}, v_{0} \in P_{h} \subset E$ and $r \in(0,1)$ such that

$$
\begin{aligned}
& r v_{0} \preceq u_{0} \prec v_{0}, \quad \text { that is, } \quad r v_{0} \leq u_{0}<v_{0}, \quad r D_{0^{+}}^{\gamma} v_{0} \leq D_{0^{+}}^{\gamma} u_{0}<D_{0^{+}}^{\gamma} v_{0} ; \\
& u_{0}(t) \leq \int_{0}^{1} G(t, s) f\left(s, u_{0}(s), D_{0^{+}}^{\gamma} v_{0}(s)\right) \mathrm{d} s+\int_{0}^{1} G(t, s) g\left(s, u_{0}(s)\right) \mathrm{d} s \\
& +\frac{\Gamma(\nu-\beta)}{\Gamma(v)} k\left(v_{0}(1)\right) t^{\nu-1}, \quad t \in[0,1] ; \\
& D_{0^{+}}^{\gamma} u_{0}(t) \leq \int_{0}^{1} D_{0^{+}}^{\gamma} G(t, s) f\left(s, u_{0}(s), D_{0^{+}}^{\gamma} \nu_{0}(s)\right) \mathrm{d} s+\int_{0}^{1} D_{0^{+}}^{\gamma} G(t, s) g\left(s, u_{0}(s)\right) \mathrm{d} s \\
& +\frac{\Gamma(\nu-\beta)}{\Gamma(v-\gamma)} k\left(v_{0}(1)\right) t^{\nu-\gamma-1}, \quad t \in[0,1] ; \\
& v_{0}(t) \geq \int_{0}^{1} G(t, s) f\left(s, v_{0}(s), D_{0^{+}}^{\gamma} u_{0}(s)\right) \mathrm{d} s+\int_{0}^{1} G(t, s) g\left(s, v_{0}(s)\right) \mathrm{d} s \\
& +\frac{\Gamma(\nu-\beta)}{\Gamma(\nu)} k\left(u_{0}(1)\right) t^{\nu-1}, \quad t \in[0,1] ; \\
& D_{0^{+}}^{\gamma} v_{0}(t) \leq \int_{0}^{1} D_{0^{+}}^{\gamma} G(t, s) f\left(s, v_{0}(s), D_{0^{+}}^{\gamma} u_{0}(s)\right) \mathrm{d} s+\int_{0}^{1} D_{0^{+}}^{\gamma} G(t, s) g\left(s, v_{0}(s)\right) \mathrm{d} s \\
& +\frac{\Gamma(v-\beta)}{\Gamma(v-\gamma)} k\left(u_{0}(1)\right) t^{\nu-\gamma-1}, \quad t \in[0,1] ;
\end{aligned}
$$

where $h(t)=t^{\nu-1}, t \in[0,1]$ and $G(t, s)$ is defined by (2.4); 
(2) the problem (1.1) has a unique positive solution $x^{*}$ in $P_{h}$;

(3) for any initial value $x_{0}, y_{0} \in P_{h}$, there are two iterative sequences $\left\{x_{n}\right\},\left\{y_{n}\right\}$ for approximating $x^{*}$, that is, $x_{n} \rightarrow x^{*}, y_{n} \rightarrow x^{*}$, as $n \rightarrow \infty$, where

$$
\begin{aligned}
x_{n}(t)= & \int_{0}^{1} G(t, s) f\left(s, x_{n-1}(s), D_{0^{+}}^{\gamma} y_{n-1}(s)\right) \mathrm{d} s+\int_{0}^{1} G(t, s) g\left(s, x_{n-1}(s)\right) \mathrm{d} s \\
& +\frac{\Gamma(v-\beta)}{\Gamma(v)} k\left(y_{n-1}(1)\right) t^{\nu-1}, \quad n=1,2, \ldots ; \\
y_{n}(t)= & \int_{0}^{1} G(t, s) f\left(s, y_{n-1}(s), D_{0^{+}}^{\gamma} x_{n-1}(s)\right) \mathrm{d} s+\int_{0}^{1} G(t, s) g\left(s, y_{n-1}(s)\right) \mathrm{d} s \\
& +\frac{\Gamma(v-\beta)}{\Gamma(v)} k\left(x_{n-1}(1)\right) t^{\nu-1}, \quad n=1,2, \ldots
\end{aligned}
$$

Proof By (3.1)-(3.3), we can calculate that

$$
\begin{aligned}
& D_{0^{+}}^{\gamma} A(u)(t)=\int_{0}^{1} D_{0^{+}}^{\gamma} G(t, s) g(s, u(s)) \mathrm{d} s, \quad t \in[0,1] ; \\
& D_{0^{+}}^{\gamma} B(v)(t)=\frac{\Gamma(v-\beta)}{\Gamma(\nu-\gamma)} k(v(1)) t^{\nu-\gamma-1}, \quad t \in[0,1] ; \\
& D_{0^{+}}^{\gamma} C(u, v)(t)=\int_{0}^{1} D_{0^{+}}^{\gamma} G(t, s) f\left(s, u(s), D_{0^{+}}^{\gamma} v(s)\right) \mathrm{d} s, \quad t \in[0,1] .
\end{aligned}
$$

To get the conclusions, we need to prove that the operators $A, B, C$ satisfies all conditions in Lemma 2.5.

Firstly, the proof of $A: P \rightarrow P, B: P \rightarrow P$ and $C: P \times P \rightarrow P$ is presented. $\forall u, v \in P$, from $\left(H_{1}\right)$ and Lemma 2.4 , it is easy to obtain $A(u)(t) \geq 0, D_{0^{+}}^{\gamma} A(u)(t) \geq 0, B(v)(t) \geq 0$, $D_{0^{+}}^{\gamma} B(v)(t) \geq 0, C(u, v)(t) \geq 0, D_{0^{+}}^{\gamma} C(u, v)(t) \geq 0, \forall t \in[0,1]$. That is, $A(u) \in P, B(v) \in P$, $C(u, v) \in P$. Therefore, $A: P \rightarrow P, B: P \rightarrow P$ and $C: P \times P \rightarrow P$. Next, we show $A$ is increasing and $B$ is decreasing. $\forall u, v \in P$ and $u \preceq v$, we know that $u(t) \leq v(t), D_{0^{+}}^{\gamma} u(t) \leq D_{0^{+}}^{\gamma} v(t)$, $\forall t \in[0,1]$. From $\left(H_{2}\right)$ and $G(t, s) \succeq 0$, we have

$$
\begin{aligned}
& A(u)=\int_{0}^{1} G(t, s) g(s, u(s)) \mathrm{d} s \leq \int_{0}^{1} G(t, s) g(s, v(s)) \mathrm{d} s=A(v) ; \\
& D_{0^{+}}^{\gamma} A(u)=\int_{0}^{1} D_{0^{+}}^{\gamma} G(t, s) g(s, u(s)) \mathrm{d} s \leq \int_{0}^{1} D_{0^{+}}^{\gamma} G(t, s) g(s, v(s)) \mathrm{d} s=D_{0^{+}}^{\gamma} A(v) ; \\
& B(u)=\frac{\Gamma(v-\beta)}{\Gamma(v)} k(u(1)) t^{\nu-1} \geq \frac{\Gamma(v-\beta)}{\Gamma(v)} k(v(1)) t^{\nu-1}=B(v) ; \\
& D_{0^{+}}^{\gamma} B(u)=\frac{\Gamma(v-\beta)}{\Gamma(v-\gamma)} k(u(1)) t^{\nu-\gamma-1} \geq \frac{\Gamma(v-\beta)}{\Gamma(v-\gamma)} k(v(1)) t^{\nu-\gamma-1}=D_{0^{+}}^{\gamma} B(v) .
\end{aligned}
$$

Hence, $A(u) \preceq A(v)$ and $B(u) \succeq B(v)$.

Further, we prove that $C$ is a mixed monotone operator. $\forall u_{1}, u_{2}, v_{1}, v_{2} \in P$ with $u_{1} \preceq u_{2}$, $v_{1} \succeq v_{2}$, that is, $u_{1}(t) \leq u_{2}(t), D_{0^{+}}^{\gamma} u_{1}(t) \leq D_{0^{+}}^{\gamma} u_{2}(t), v_{1}(t) \geq u_{2}(t), D_{0^{+}}^{\gamma} v_{1}(t) \geq D_{0^{+}}^{\gamma} v_{2}(t), t \in$ 
$[0,1]$. By $\left(H_{2}\right)$ and $G(t, s) \succeq 0$, we get

$$
\begin{aligned}
& C\left(u_{1}, v_{1}\right)=\int_{0}^{1} G(t, s) f\left(s, u_{1}(s), D_{0^{+}}^{\gamma} v_{1}(s)\right) \mathrm{d} s \\
& \quad \leq \int_{0}^{1} G(t, s) f\left(s, u_{2}(s), D_{0^{+}}^{\gamma} v_{2}(s)\right) \mathrm{d} s=C\left(u_{2}, v_{2}\right), \\
& \begin{aligned}
D_{0^{+}}^{\gamma} C\left(u_{1}, v_{1}\right) & =\int_{0}^{1} D_{0^{+}}^{\gamma} G(t, s) f\left(s, u_{1}(s), D_{0^{+}}^{\gamma} v_{1}(s)\right) \mathrm{d} s \\
& \leq \int_{0}^{1} D_{0^{+}}^{\gamma} G(t, s) f\left(s, u_{2}(s), D_{0^{+}}^{\gamma} v_{2}(s)\right) \mathrm{d} s=D_{0^{+}}^{\gamma} C\left(u_{2}, v_{2}\right) .
\end{aligned}
\end{aligned}
$$

Thus, $C\left(u_{1}, v_{1}\right) \preceq C\left(u_{2}, v_{2}\right)$.

Now we prove $A$ satisfies (2.13) and $B$ and $C$ satisfy (2.14). From $\left(H_{3}\right), \eta \in(0,1)$, we obtain

$$
\begin{aligned}
& A(\eta x)=\int_{0}^{1} G(t, s) g(s, \eta x(s)) \mathrm{d} s \geq \eta \int_{0}^{1} G(t, s) g(s, x(s)) \mathrm{d} s=\eta A(x), \\
& D_{0^{+}}^{\gamma} A(\eta x)=\int_{0}^{1} D_{0^{+}}^{\gamma} G(t, s) g(s, \eta x(s)) \mathrm{d} s \geq \eta \int_{0}^{1} D_{0^{+}}^{\gamma} G(t, s) g(s, x(s)) \mathrm{d} s=\eta D_{0^{+}}^{\gamma} A(x),
\end{aligned}
$$

that is, $A(t x) \succeq t A x, x \in P$. Then $A$ is a sub-homogeneous operator. Clearly, the following inequalities are also tenable:

$$
\begin{aligned}
& B\left(\eta^{-1} y\right)=\frac{\Gamma(\nu-\beta)}{\Gamma(\nu)} k\left(\eta^{-1} y(1)\right) t^{\nu-1} \geq \eta \frac{\Gamma(\nu-\beta)}{\Gamma(v)} k(y(1)) t^{\nu-1}=\eta B(y), \\
& D_{0^{+}}^{\gamma} B\left(\eta^{-1} y\right)=\frac{\Gamma(\nu-\beta)}{\Gamma(v-\gamma)} k\left(\eta^{-1} y(1)\right) t^{\nu-\gamma-1} \geq \eta \frac{\Gamma(\nu-\beta)}{\Gamma(v-\gamma)} k(y(1)) t^{\nu-\gamma-1}=\eta D_{0^{+}}^{\gamma} B(y), \\
& C\left(\eta x, \eta^{-1} y\right)=\int_{0}^{1} G(t, s) f\left(s, \eta x(s), \eta^{-1} D_{0^{+}}^{\gamma} y(s)\right) \mathrm{d} s \\
& \geq \eta^{\alpha} \int_{0}^{1} G(t, s) f\left(s, x(s), D_{0^{+}}^{\gamma} y(s)\right) \mathrm{d} s=\eta^{\alpha} C(x, y), \\
& D_{0^{+}}^{\gamma} C\left(\eta x, \eta^{-1} y\right)=\int_{0}^{1} D_{0^{+}}^{\gamma} G(t, s) f\left(s, \eta x(s), \eta^{-1} D_{0^{+}}^{\gamma} y(s)\right) \mathrm{d} s \\
& \geq \eta^{\alpha} \int_{0}^{1} D_{0^{+}}^{\gamma} G(t, s) f\left(s, x(s), D_{0^{+}}^{\gamma} y(s)\right) \mathrm{d} s=\eta^{\alpha} D_{0^{+}}^{\gamma} C(x, y) .
\end{aligned}
$$

Hence, $B\left(t^{-1} y\right) \succeq t B y$ and $C(x, y) \succeq t^{\alpha} C(x, y)$.

In the sequel, we demonstrate that the assumptions $\left(A_{1}\right)$ and $\left(A_{2}\right)$ are satisfied. In view of $\left(H_{1}\right),\left(H_{2}\right)$ and $(2.9)$ in Lemma 2.4, we have

$$
\begin{aligned}
A(h) & =\int_{0}^{1} G(t, s) g\left(s, s^{\nu-1}\right) \mathrm{d} s \\
& \leq \int_{0}^{1} \frac{t^{\nu-1}(1-s)^{\nu-\beta-1}}{\Gamma(\nu)} g\left(s, s^{\nu-1}\right) \mathrm{d} s \\
& \leq t^{\nu-1} \int_{0}^{1} \frac{(1-s)^{\nu-\beta-1} g(s, 1)}{\Gamma(\nu)} \mathrm{d} s,
\end{aligned}
$$




$$
\begin{aligned}
A(h) \geq & \int_{0}^{1} \frac{t^{\nu-1}(1-s)^{\nu-\beta-1}\left[1-(1-s)^{\beta}\right]}{\Gamma(\nu)} g\left(s, s^{\nu-1}\right) \mathrm{d} s \\
& \geq t^{\nu-1} \int_{0}^{1} \frac{(1-s)^{\nu-\beta-1}\left[1-(1-s)^{\beta}\right] g(s, 0)}{\Gamma(\nu)} \mathrm{d} s, \\
C(h, h) & =\int_{0}^{1} G(t, s) f\left(s, s^{\nu-1}, D_{0^{+}}^{\gamma} s^{\nu-1}\right) \mathrm{d} s \\
& \leq \int_{0}^{1} \frac{t^{\nu-1}(1-s)^{\nu-\beta-1}}{\Gamma(\nu)} f\left(s, s^{\nu-1}, \frac{\Gamma(v)}{\Gamma(\nu-\gamma)} t^{\nu-\gamma-1}\right) \mathrm{d} s \\
& \leq t^{\nu-1} \int_{0}^{1} \frac{(1-s)^{\nu-\beta-1}}{\Gamma(\nu)} f(s, 1,0) \mathrm{d} s, \\
C(h, h) & \geq \int_{0}^{1} \frac{t^{\nu-1}(1-s)^{\nu-\beta-1}\left[1-(1-s)^{\beta}\right]}{\Gamma(\nu)} f\left(s, s^{\nu-1}, \frac{\Gamma(\nu)}{\Gamma(\nu-\gamma)} t^{\nu-\gamma-1}\right) \mathrm{d} s \\
& \geq t^{\nu-1} \int_{0}^{1} \frac{(1-s)^{\nu-\beta-1}\left[1-(1-s)^{\beta}\right]}{\Gamma(\nu)} f\left(s, 0, \frac{\Gamma(\nu)}{\Gamma(\nu-\gamma)}\right) \mathrm{d} s .
\end{aligned}
$$

On the other hand, $\left(H_{1}\right),\left(H_{2}\right)$ and (2.10) imply

$$
\begin{aligned}
& D_{0+}^{\gamma} A(h)=\int_{0}^{1} D_{0+}^{\gamma} G(t, s) g\left(s, s^{\nu-1}\right) \mathrm{d} s \\
& \leq \int_{0}^{1} \frac{t^{\nu-\gamma-1}(1-s)^{\nu-\beta-1}}{\Gamma(\nu-\gamma)} g\left(s, s^{\nu-1}\right) \mathrm{d} s \\
& \leq \frac{\Gamma(v)}{\Gamma(v-\gamma)} t^{\nu-\gamma-1} \int_{0}^{1} \frac{(1-s)^{\nu-\beta-1} g(s, 1)}{\Gamma(v)} \mathrm{d} s, \\
& D_{0+}^{\gamma} A(h) \geq \int_{0}^{1} \frac{t^{\nu-\gamma-1}(1-s)^{\nu-\beta-1}\left[1-(1-s)^{\beta-\gamma}\right]}{\Gamma(\nu-\gamma)} g\left(s, s^{\nu-1}\right) \mathrm{d} s \\
& \geq \frac{\Gamma(v)}{\Gamma(v-\gamma)} t^{\nu-\gamma-1} \int_{0}^{1} \frac{(1-s)^{\nu-\beta-1}\left[1-(1-s)^{\beta-\gamma}\right] g(s, 0)}{\Gamma(v)} \mathrm{d} s, \\
& D_{0+}^{\gamma} C(h, h)=\int_{0}^{1} D_{0+}^{\gamma} G(t, s) f\left(s, s^{\nu-1}, D_{0^{+}}^{\gamma} s^{\nu-1}\right) \mathrm{d} s \\
& \leq \int_{0}^{1} \frac{t^{\nu-\gamma-1}(1-s)^{\nu-\beta-1}}{\Gamma(\nu-\gamma)} f\left(s, s^{\nu-1}, \frac{\Gamma(\nu)}{\Gamma(\nu-\gamma)} t^{\nu-\gamma-1}\right) \mathrm{d} s \\
& \leq \frac{\Gamma(v)}{\Gamma(v-\gamma)} t^{\nu-\gamma-1} \int_{0}^{1} \frac{(1-s)^{\nu-\beta-1}}{\Gamma(v)} f(s, 1,0) \mathrm{d} s, \\
& D_{0+}^{\gamma} C(h, h) \geq \int_{0}^{1} \frac{t^{\nu-1}(1-s)^{\nu-\beta-1}\left[1-(1-s)^{\beta-\gamma}\right]}{\Gamma(v)} f\left(s, s^{\nu-1}, \frac{\Gamma(v)}{\Gamma(v-\gamma)} t^{\nu-\gamma-1}\right) \mathrm{d} s \\
& \geq \frac{\Gamma(v)}{\Gamma(v-\gamma)} t^{\nu-\gamma-1} \int_{0}^{1} \frac{(1-s)^{\nu-\beta-1}\left[1-(1-s)^{\beta-\gamma}\right]}{\Gamma(\nu)} f\left(s, 0, \frac{\Gamma(v)}{\Gamma(v-\gamma)}\right) \mathrm{d} s .
\end{aligned}
$$

Set

$$
\begin{aligned}
& c_{1}=\int_{0}^{1} \frac{(1-s)^{\nu-\beta-1}\left[1-(1-s)^{\beta-\gamma}\right] g(s, 0)}{\Gamma(v)} \mathrm{d} s, \\
& c_{2}=\int_{0}^{1} \frac{(1-s)^{\nu-\beta-1} g(s, 1)}{\Gamma(\nu)} \mathrm{d} s,
\end{aligned}
$$




$$
\begin{aligned}
& c_{3}=\int_{0}^{1} \frac{(1-s)^{\nu-\beta-1}\left[1-(1-s)^{\beta-\gamma}\right] f\left(s, 0, \frac{\Gamma(\nu)}{\Gamma(\nu-\gamma)}\right)}{\Gamma(\nu)} \mathrm{d} s, \\
& c_{4}=\int_{0}^{1} \frac{(1-s)^{\nu-\beta-1}}{\Gamma(\nu)} f(s, 1,0) \mathrm{d} s .
\end{aligned}
$$

From $\left(H_{2}\right)$ and $\left(H_{4}\right)$, it is clear that

$$
c_{2} \geq c_{1}>0, \quad c_{4} \geq c_{3} \geq \delta_{1} c_{1}>0 .
$$

Consequently,

$$
c_{1} h \preceq A(h) \preceq c_{2} h, \quad c_{3} h \preceq C(h, h) \preceq c_{4} h .
$$

Further,

$$
\begin{aligned}
& B(h)=t^{\nu-1} \frac{\Gamma(v-\beta)}{\Gamma(v)} k(1)=\frac{\Gamma(v-\beta)}{\Gamma(v)} k(1) h(t), \\
& D_{0^{+}}^{\gamma} B(h)=\frac{\Gamma(v)}{\Gamma(v-\gamma)} t^{\nu-\gamma-1} \frac{\Gamma(v-\beta)}{\Gamma(v)} k(1)=\frac{\Gamma(v-\beta)}{\Gamma(v)} k(1) D_{0^{+}}^{\gamma} h(t) .
\end{aligned}
$$

From $k(y(1)) \neq 0$, we have $B(h) \in P_{h}$. Therefore, $\left(A_{1}\right)$ in Lemma 2.5 is proved.

Next, we show the proof of the condition $\left(A_{2}\right)$ of Lemma 2.5. By $\left(H_{4}\right)$,

$$
\begin{aligned}
C(x, y) & =\int_{0}^{1} G(t, s) f\left(s, x(s), D_{0^{+}}^{\gamma} y(s)\right) \mathrm{d} s \\
\geq & \delta_{1} \int_{0}^{1} G(t, s) g(s, x(s)) \mathrm{d} s \\
& =\delta_{1} A x, \\
D_{0^{+}}^{\gamma} C(x, y) & =\int_{0}^{1} D_{0^{+}}^{\gamma} G(t, s) f\left(s, x(s), D_{0^{+}}^{\gamma} y(s)\right) \mathrm{d} s \\
& \geq \delta_{1} \int_{0}^{1} D_{0^{+}}^{\gamma} G(t, s) g(s, x(s)) \mathrm{d} s \\
& =\delta_{1} D_{0^{+}}^{\gamma} A x .
\end{aligned}
$$

Then

$$
C(x, y) \succeq \delta_{1} A x .
$$

From $\left(H_{4}\right)$ and Lemma 2.4 , we have

$$
\begin{aligned}
C(x, y) & =\int_{0}^{1} G(t, s) f\left(s, x(s), D_{0^{+}}^{\gamma} y(s)\right) \mathrm{d} s \\
& \geq \int_{0}^{1} \frac{t^{\nu-1}(1-s)^{\nu-\beta-1}\left[1-(1-s)^{\beta}\right]}{\Gamma(v)} f\left(s, x(s), D_{0^{+}}^{\gamma} y(s)\right) \mathrm{d} s \\
& \geq \frac{t^{\nu-1}}{\Gamma(v)}\left(\frac{1}{v-\beta}-\frac{1}{v}\right) \delta_{2}
\end{aligned}
$$




$$
\begin{aligned}
\geq & \frac{t^{\nu-1}}{\Gamma(v)}\left(\frac{1}{v-\beta}-\frac{1}{v-\gamma}\right) k(y(1)) \\
= & \frac{1}{\Gamma(v-\beta)}\left(\frac{1}{v-\beta}-\frac{1}{v-\gamma}\right) B y, \\
D_{0^{+}}^{\gamma} C(x, y) & =\int_{0}^{1} D_{0^{+}}^{\gamma} G(t, s) f\left(s, x(s), D_{0^{+}}^{\gamma} y(s)\right) \mathrm{d} s \\
& \geq \frac{t^{\nu-\gamma-1}}{\Gamma(v-\gamma)} \int_{0}^{1}(1-s)^{\nu-\beta-1}\left(1-(1-s)^{\beta-\gamma}\right) f\left(s, x(s), D_{0^{+}}^{\gamma} y(s)\right) \mathrm{d} s \\
& \geq \frac{t^{\nu-\gamma-1}}{\Gamma(v-\gamma)}\left(\frac{1}{v-\beta}-\frac{1}{v-\gamma}\right) k(y(1)) \\
& =\frac{1}{\Gamma(v-\beta)}\left(\frac{1}{v-\beta}-\frac{1}{v-\gamma}\right) D_{0^{+}}^{\gamma} B y .
\end{aligned}
$$

That means $C(x, y) \succeq \frac{1}{\Gamma(\nu-\beta)}\left(\frac{1}{v-\beta}-\frac{1}{\nu-\gamma}\right) B y$. Let $\delta=\min \left\{\delta_{1}, \frac{1}{\Gamma(\nu-\beta)}\left(\frac{1}{v-\beta}-\frac{1}{\nu-\gamma}\right)\right\}$. Then we have

$$
C(x, y) \succeq \delta(A x+B y) .
$$

Finally, as an application of Lemma 2.5, we see that the conclusion (2) in Lemma 2.5 means that there exist $u_{0}, v_{0} \in P_{h}$ and $r \in(0,1)$ such that

$$
\begin{aligned}
& r v_{0} \leq u_{0} \prec v_{0}, \quad \text { that is, } \quad r v_{0} \leq u_{0}<v_{0}, \quad r D_{0^{+}}^{\gamma} v_{0} \leq D_{0^{+}}^{\gamma} u_{0}<D_{0^{+}}^{\gamma} v_{0} ; \\
& \begin{aligned}
& u_{0}(t) \leq \int_{0}^{1} G(t, s) f\left(s, u_{0}(s), D_{0^{+}}^{\gamma} v_{0}(s)\right) \mathrm{d} s+\int_{0}^{1} G(t, s) g\left(s, u_{0}(s)\right) \mathrm{d} s \\
&+\frac{\Gamma(v-\beta)}{\Gamma(v)} k\left(v_{0}(1)\right) t^{\nu-1}, \quad t \in[0,1] ; \\
& D_{0^{+}}^{\gamma} u_{0}(t) \leq \int_{0}^{1} D_{0^{+}}^{\gamma} G(t, s) f\left(s, u_{0}(s), D_{0^{+}}^{\gamma} \nu_{0}(s)\right) \mathrm{d} s+\int_{0}^{1} D_{0^{+}}^{\gamma} G(t, s) g\left(s, u_{0}(s)\right) \mathrm{d} s \\
&+\frac{\Gamma(v-\beta)}{\Gamma(v-\gamma)} k\left(v_{0}(1)\right) t^{\nu-\gamma-1}, \quad t \in[0,1] ; \\
& v_{0}(t) \geq \int_{0}^{1} G(t, s) f\left(s, v_{0}(s), D_{0^{+}}^{\gamma} u_{0}(s)\right) \mathrm{d} s+\int_{0}^{1} G(t, s) g\left(s, v_{0}(s)\right) \mathrm{d} s \\
&+\frac{\Gamma(v-\beta)}{\Gamma(v)} k\left(u_{0}(1)\right) t^{\nu-1}, \quad t \in[0,1] ; \\
& D_{0^{+}}^{\gamma} v_{0}(t) \leq \int_{0}^{1} D_{0^{+}}^{\gamma} G(t, s) f\left(s, v_{0}(s), D_{0^{+}}^{\gamma} u_{0}(s)\right) \mathrm{d} s+\int_{0}^{1} D_{0^{+}}^{\gamma} G(t, s) g\left(s, v_{0}(s)\right) \mathrm{d} s \\
&+\frac{\Gamma(v-\beta)}{\Gamma(v-\gamma)} k\left(u_{0}(1)\right) t^{\nu-\gamma-1}, \quad t \in[0,1] .
\end{aligned}
\end{aligned}
$$

Equation (3) in Lemma 2.5 implies that the problem (1.1) has a unique positive solution $x^{*}$ in $P_{h}$; Lemma 2.5(4) means that, for any initial value $x_{0}, y_{0} \in P_{h}$, there are two iterative sequences $\left\{x_{n}\right\},\left\{y_{n}\right\}$ for approximating $x^{*}$, that is, $x_{n} \rightarrow x^{*}, y_{n} \rightarrow x^{*}$, as $n \rightarrow \infty$, where

$$
\begin{aligned}
x_{n}(t)= & \int_{0}^{1} G(t, s) f\left(s, x_{n-1}(s), D_{0^{+}}^{\gamma} y_{n-1}(s)\right) \mathrm{d} s+\int_{0}^{1} G(t, s) g\left(s, x_{n-1}(s)\right) \mathrm{d} s \\
& +\frac{\Gamma(v-\beta)}{\Gamma(v)} k\left(y_{n-1}(1)\right) t^{\nu-1}, \quad n=1,2, \ldots ;
\end{aligned}
$$




$$
\begin{aligned}
y_{n}(t)= & \int_{0}^{1} G(t, s) f\left(s, y_{n-1}(s), D_{0^{+}}^{\gamma} x_{n-1}(s)\right) \mathrm{d} s+\int_{0}^{1} G(t, s) g\left(s, y_{n-1}(s)\right) \mathrm{d} s \\
& +\frac{\Gamma(v-\beta)}{\Gamma(v)} k\left(x_{n-1}(1)\right) t^{\nu-1}, \quad n=1,2, \ldots
\end{aligned}
$$

Theorem 3.2 Assume $\left(H_{1}\right),\left(H_{2}\right)$ and suppose that

$\left(H_{3}^{\prime}\right)$ there exists a constant $\alpha \in(0,1)$ such that $k\left(\eta^{-1} y\right) \geq \eta^{\alpha} k(y), \forall \eta \in(0,1), t \in[0,1], y \in$ $[0,+\infty)$ And $g(t, x), f(t, x, y)$ satisfy $g(t, \eta x) \geq \eta g(t, x), f\left(t, \eta x, \eta^{-1} y\right) \geq \eta f(t, x, y), \forall \eta \in$ $(0,1), x, y \in[0,+\infty)$.

$\left(H_{4}^{\prime}\right) g(t, 0) \not \equiv 0, f\left(t, 0, \frac{\Gamma(v)}{\Gamma(v-\beta)}\right) \not \equiv 0$ for $t \in[0,1]$ and there exists a constant $\delta_{0}$ such that $g(t, x)+f(t, x, y) \leq \delta_{0} \leq k(y), t \in[0,1], x, y \in[0,+\infty)$.

Then the conclusions (1)-(3) of Theorem 3.1 hold.

Proof Similar to the proof of Theorem 3.1, from $\left(H_{1}\right)$ and $\left(H_{2}\right)$, we see that $A, B: P \rightarrow P$, $C: P \times P \rightarrow P$ and $A$ is increasing, $B$ is decreasing, $C$ is a mixed monotone operator. From $\left(H_{3}^{\prime}\right)$, we have

$$
\begin{aligned}
& A(\eta x)=\int_{0}^{1} G(t, s) g(s, \eta x(s)) \mathrm{d} s \geq \eta \int_{0}^{1} G(t, s) g(s, x(s)) \mathrm{d} s=\eta A(x), \\
& D_{0^{+}}^{\gamma} A(\eta x)=\int_{0}^{1} D_{0^{+}}^{\gamma} G(t, s) g(s, \eta x(s)) \mathrm{d} s \geq \eta \int_{0}^{1} D_{0^{+}}^{\gamma} G(t, s) g(s, x(s)) \mathrm{d} s=\eta D_{0^{+}}^{\gamma} A(x), \\
& B\left(\eta^{-1} y\right)=\frac{\Gamma(v-\beta)}{\Gamma(v)} k\left(\eta^{-1} y(1)\right) t^{\nu-1} \geq \eta^{\alpha} \frac{\Gamma(\nu-\beta)}{\Gamma(v)} k(y(1)) t^{\nu-1}=\eta^{\alpha} B(y), \\
& D_{0^{+}}^{\gamma} B\left(\eta^{-1} u\right)=\frac{\Gamma(v-\beta)}{\Gamma(v-\gamma)} k\left(\eta^{-1} y(1)\right) t^{\nu-\gamma-1} \geq \eta^{\alpha} \frac{\Gamma(v-\beta)}{\Gamma(v-\gamma)} k(y(1)) t^{\nu-\gamma-1}=\eta^{\alpha} D_{0^{+}}^{\gamma} B(y), \\
& C\left(\eta x, \eta^{-1} y\right)=\int_{0}^{1} G(t, s) f\left(s, \eta x(s), \eta^{-1} D_{0^{+}}^{\gamma} y(s)\right) \mathrm{d} s \\
& \geq \eta \int_{0}^{1} G(t, s) f\left(s, x(s), D_{0^{+}}^{\gamma} y(s)\right) \mathrm{d} s=\eta C(x, y), \\
& D_{0^{+}}^{\gamma} C\left(\eta x, \eta^{-1} \eta y\right)=\int_{0}^{1} D_{0^{+}}^{\gamma} G(t, s) f\left(s, \eta(s), \eta^{-1} D_{0^{+}}^{\gamma} y(s)\right) \mathrm{d} s \\
& \geq \eta \int_{0}^{1} D_{0^{+}}^{\gamma} G(t, s) f\left(s, x(s), D_{0^{+}}^{\gamma} y(s)\right) \mathrm{d} s=\eta D_{0^{+}}^{\gamma} C(x, y) .
\end{aligned}
$$

Hence, $A(t x) \succeq t A x, B\left(t^{-1} y\right) \succeq t^{\alpha} B y$ and $C(x, y) \succeq t C(x, y)$. Then $A$ is a sub-homogeneous operator. Since $f\left(t, 0, \frac{\Gamma(v)}{\Gamma(\nu-\beta)}\right) \not \equiv 0$ and by $\left(H_{2}\right)$, we obtain $c_{4} \geq c_{3}>0$. Obviously, $C(h, h) \in$ $P_{h}$. From $g(t, 0) \not \equiv 0$ and $\left(H_{2}\right)$, we have $c_{2} \geq c_{1}>0$, which implies $A(h) \in P_{h}$. Here, $c_{1}, c_{2}$, $c_{3}, c_{4}$ is given by (3.7), (3.8). From (3.9) and (3.10) in Theorem 3.1 and $k(y(1)) \neq 0$, we get $B(h) \in P_{h}$.

Now, we show the assumption $\left(A_{2}^{\prime}\right)$ is satisfied. From $\left(H_{4}^{\prime}\right)$ and Lemma 2.4, for $t \in(0,1)$, $x, y \in[0,+\infty)$, we obtain

$$
\begin{aligned}
A x+C(x, y) & =\int_{0}^{1} G(t, s)\left(g(s, x(s))+f\left(s, x(s), D_{0^{+}}^{\gamma} y(s)\right)\right) \mathrm{d} s \\
& \leq \int_{0}^{1} \frac{t^{\nu-1}(1-s)^{\nu-\beta-1}}{\Gamma(\nu)}\left(g(s, x(s))+f\left(s, x(s), D_{0^{+}}^{\gamma} y(s)\right)\right) \mathrm{d} s
\end{aligned}
$$




$$
\begin{aligned}
& \leq \int_{0}^{1} \frac{t^{\nu-1}(1-s)^{\nu-\beta-1}}{\Gamma(v)} \delta_{0} \mathrm{~d} s \\
& \leq \frac{1}{(v-\beta) \Gamma(v-\beta)} \frac{\Gamma(\nu-\beta)}{\Gamma(v)} k(y(1)) t^{\nu-1} \\
& =\frac{1}{\Gamma(v-\beta+1)} B y, \\
D_{0^{+}}^{\gamma}(A x+C(x, y)) & =\int_{0}^{1} D_{0^{+}}^{\gamma} G(t, s)\left(g(s, x(s))+f\left(s, x(s), D_{0^{+}}^{\gamma} y(s)\right) \mathrm{d} s\right. \\
& \leq \int_{0}^{1} \frac{t^{\nu-\gamma-1}(1-s)^{\nu-\beta-1}}{\Gamma(v-\gamma)}\left(g(s, x(s))+f\left(s, x(s), D_{0^{+}}^{\gamma} y(s)\right) \mathrm{d} s\right. \\
& \leq \int_{0}^{1} \frac{t^{\nu-\gamma-1}(1-s)^{\nu-\beta-1}}{\Gamma(v-\gamma)} \delta_{0} \mathrm{~d} s \\
& \leq \frac{1}{(v-\beta) \Gamma(v-\beta)} \frac{\Gamma(v-\beta)}{\Gamma(v-\gamma)} k(y(1)) t^{\nu-\gamma-1} \\
& =\frac{1}{\Gamma(v-\beta+1)} D_{0^{+}}^{\gamma} B y .
\end{aligned}
$$

Let $\delta=\frac{1}{\Gamma(v-\beta+1)}$. Then

$$
A x+C(x, y) \preceq \delta B y .
$$

Therefore, by Lemma 2.6, the conclusions (1)-(3) in Theorem 3.1 hold.

Theorem 3.3 Assume $\left(H_{1}\right),\left(H_{2}\right)$ and suppose that

$\left(H_{3}^{\prime \prime}\right)$ there exists a constant $\alpha \in(0,1)$ such that $g(t, \eta x) \geq \eta^{\alpha} g(t, x), \forall \eta \in(0,1), t \in[0,1]$, $x \in[0,+\infty)$. And $k(y), f(t, x, y)$ satisfy $k\left(\eta^{-1} y\right) \geq \eta k(y), f\left(t, \eta x, \eta^{-1} y\right) \geq \eta f(t, x, y), \forall \eta \in$ $(0,1), x, y \in[0,+\infty)$.

$\left(H_{4}^{\prime \prime}\right) f\left(t, 0, \frac{\Gamma(v)}{\Gamma(v-\beta)}\right) \not \equiv 0$ for $t \in[0,1], k(y(1)) \neq 0$ and there exist two constants $\delta_{1}, \delta_{2}$ such that $k(y) \leq \delta_{1} \leq g(t, x)$ and $f(t, x, y) \leq \delta_{2} g(t, x), t \in[0,1], x, y \in[0,+\infty)$.

Then the conclusions (1)-(3) of Theorem 3.1 hold.

Proof From the proof of Theorem 3.1, we know that $A: P \rightarrow P$ is an increasing operator, $B: P \rightarrow P$ is a decreasing operator, $C: P \times P \rightarrow P$ is a mixed monotone operator.

In the following, we demonstrate that $A, B, C$ satisfy all assumptions in Lemma 2.7. From $\left(H_{3}^{\prime \prime}\right)$, we know that

$$
\begin{aligned}
& A(\eta x)=\int_{0}^{1} G(t, s) g(s, \eta x(s)) \mathrm{d} s \geq \eta^{\alpha} \int_{0}^{1} G(t, s) g(s, x(s)) \mathrm{d} s=\eta^{\alpha} A(x), \\
& D_{0^{+}}^{\gamma} A(\eta x)=\int_{0}^{1} D_{0^{+}}^{\gamma} G(t, s) g(s, \eta x(s)) \mathrm{d} s \geq \eta^{\alpha} \int_{0}^{1} D_{0^{+}}^{\gamma} G(t, s) g(s, x(s)) \mathrm{d} s=\eta D_{0^{+}}^{\gamma} A(x), \\
& B\left(\eta^{-1} y\right)=\frac{\Gamma(v-\beta)}{\Gamma(v)} k\left(\eta^{-1} y(1)\right) t^{\nu-1} \geq \eta \frac{\Gamma(v-\beta)}{\Gamma(v)} k(y(1)) t^{\nu-1}=\eta B(y), \\
& D_{0^{+}}^{\gamma} B\left(\eta^{-1} y\right)=\frac{\Gamma(v-\beta)}{\Gamma(v-\gamma)} k\left(\eta^{-1} y(1)\right) t^{\nu-\gamma-1} \geq \eta \frac{\Gamma(v-\beta)}{\Gamma(v-\gamma)} k(y(1)) t^{\nu-\gamma-1}=\eta D_{0^{+}}^{\gamma} B(y),
\end{aligned}
$$




$$
\begin{aligned}
& C\left(\eta x, \eta^{-1} y\right)=\int_{0}^{1} G(t, s) f\left(s, \eta x(s), \eta^{-1} D_{0^{+}}^{\gamma} y(s)\right) \mathrm{d} s \\
& \geq \eta \int_{0}^{1} G(t, s) f\left(s, x(s), D_{0^{+}}^{\gamma} y(s)\right) \mathrm{d} s=\eta C(x, y), \\
& \begin{aligned}
D_{0^{+}}^{\gamma} C\left(\eta x, \eta^{-1} y\right) & =\int_{0}^{1} D_{0^{+}}^{\gamma} G(t, s) f\left(s, \eta(s), \eta^{-1} D_{0^{+}}^{\gamma} y(s)\right) \mathrm{d} s \\
& \geq \eta \int_{0}^{1} D_{0^{+}}^{\gamma} G(t, s) f\left(s, x(s), D_{0^{+}}^{\gamma} y(s)\right) \mathrm{d} s=\eta D_{0^{+}}^{\gamma} C(x, y) .
\end{aligned}
\end{aligned}
$$

That is, $A(t x) \succeq t^{\alpha} A x, B\left(t^{-1} y\right) \succeq t B y$ and $C(x, y) \succeq t C(x, y)$. Then $A$ is a $\alpha$-concave operator. From $\left(H_{2}\right)$, $\left(H_{4}^{\prime \prime}\right)$, we obtain $c_{4} \geq c_{3}>0$ and $c_{1} \geq c_{3}>0$, where $c_{1}, c_{2}, c_{3}, c_{4}$ are defined by (3.7), (3.8). It is evident that $C(h, h) \in P_{h}$ and $A(h) \in P_{h}$. From the proof of Theorem 3.1, we get $B(h) \in P_{h}$. Therefore, the condition $\left(A_{1}^{\prime \prime}\right)$ in Lemma 2.7 is proved.

Next, we present the assumption $\left(A_{2}^{\prime \prime}\right)$. From $\left(H_{4}^{\prime \prime}\right)$ and Lemma 2.4, for $t \in(0,1), x, y \in$ $[0,+\infty)$, we obtain

$$
\begin{aligned}
& C(x, y)=\int_{0}^{1} G(t, s) f\left(s, x(s), D_{0^{+}}^{\gamma} y(s)\right) \mathrm{d} s \\
& \leq \delta_{2} \int_{0}^{1} G(t, s) g(s, x(s)) \mathrm{d} s \\
& =\delta_{2} A x, \\
& D_{0^{+}}^{\gamma} C(x, y)=\int_{0}^{1} D_{0^{+}}^{\gamma} G(t, s) f\left(s, x(s), D_{0^{+}}^{\gamma} y(s) \mathrm{d} s\right. \\
& \leq \delta_{2} \int_{0}^{1} D_{0^{+}}^{\gamma} G(t, s) g(s, x(s)) \mathrm{d} s \\
& =\delta_{2} D_{0^{+}}^{\gamma} A x .
\end{aligned}
$$

On the other side, from $\left(H_{4}^{\prime \prime}\right)$ and Lemma 2.4 ,

$$
\begin{aligned}
A(x) & =\int_{0}^{1} G(t, s) g(s, x(s)) \mathrm{d} s \\
\geq & \int_{0}^{1} \frac{t^{\nu-1}(1-s)^{\nu-\beta-1}\left[1-(1-s)^{\beta}\right]}{\Gamma(v)} g(s, x(s)) \mathrm{d} s \\
& \geq \delta_{1} \int_{0}^{1} \frac{t^{\nu-1}(1-s)^{\nu-\beta-1}\left[1-(1-s)^{\beta}\right]}{\Gamma(v)} \mathrm{d} s \\
\geq & \frac{1}{\Gamma(v-\beta)}\left(\frac{1}{v-\beta}-\frac{1}{v}\right) \frac{\Gamma(v-\beta)}{\Gamma(v)} k(y(1)) t^{\nu-1} \\
\geq & \frac{1}{\Gamma(v-\beta)}\left(\frac{1}{v-\beta}-\frac{1}{v-\gamma}\right) \frac{\Gamma(v-\beta)}{\Gamma(\nu)} k(y(1)) t^{\nu-1} \\
& =\frac{1}{\Gamma(v-\beta)}\left(\frac{1}{v-\beta}-\frac{1}{v-\gamma}\right) B y, \\
D_{0^{+}}^{\gamma} A(x) & =D_{0^{+}}^{\gamma} \int_{0}^{1} G(t, s) g(s, x(s)) \mathrm{d} s \\
& \geq \int_{0}^{1} \frac{\left.t^{\nu-\gamma-1}(1-s)^{\nu-\beta-1}\left[1-(1-s)^{\beta-\gamma}\right)\right]}{\Gamma(\nu-\gamma)} g(s, x(s)) \mathrm{d} s
\end{aligned}
$$




$$
\begin{aligned}
& \geq \delta_{1} \int_{0}^{1} \frac{t^{\nu-\gamma-1}(1-s)^{\nu-\beta-1}\left[1-(1-s)^{\beta-\gamma}\right]}{\Gamma(\nu-\gamma)} \mathrm{d} s \\
& \geq \frac{1}{\Gamma(v-\beta)}\left(\frac{1}{v-\beta}-\frac{1}{v-\gamma}\right) \frac{\Gamma(v-\beta)}{\Gamma(v-\gamma)} k(y(1)) t^{\nu-\gamma-1} \\
& =\frac{1}{\Gamma(v-\beta)}\left(\frac{1}{v-\beta}-\frac{1}{v-\gamma}\right) D_{0^{+}}^{\gamma} B y
\end{aligned}
$$

That is, $C(x, y) \preceq \delta_{2} A x$ and $B y \preceq \frac{(\nu-\gamma) \Gamma(\nu-\beta+1)}{\beta-\gamma} A x$. Let $\delta=2 \max \left\{\delta_{2}, \frac{(\nu-\gamma) \Gamma(v-\beta+1)}{\beta-\gamma}\right\}$. Then

$$
B y+C(x, y) \preceq \delta A x .
$$

Therefore, applying Lemma 2.7, the results (1)-(3) in Theorem 3.1 are tenable.

Remark 3.1 Comparing Theorem 3.1, Theorem 3.2 and Theorem 3.3 with the main results in [9], we provide some alternative approaches to the study of the same type of problems (when the parameter $k(x)=0$ and $\gamma=0$ ) under different conditions. Our results cannot only guarantee the existence of a unique positive solution but also we obtain two sequences approximating the solution.

\section{Examples}

As applications, three examples are presented to illustrate our main results.

\subsection{Example 1}

Consider the following fractional differential equation:

$$
\left\{\begin{array}{l}
D_{0^{+}}^{\frac{9}{2}} x(t)+2(x(t))^{\frac{1}{4}}+t^{2}+\left(x^{\prime}(t)+1\right)^{-\frac{1}{2}}+1=0, \quad t \in(0,1) \\
x(0)=x^{\prime}(0)=x^{\prime \prime}(0)=x^{\prime \prime \prime}(0)=0 \\
D_{0^{+}}^{\frac{5}{2}} x(t)=\frac{1}{1+x(1)}
\end{array}\right.
$$

Let $g(t, x)=(x(t))^{\frac{1}{4}}+t^{2}$ and $g(t, 0)=t^{2} \not \equiv 0, f(t, x, y)=(x(t))^{\frac{1}{4}}+(y(t)+1)^{-\frac{1}{2}}+1$ and $k(y)=\frac{1}{1+y}$. Clearly, $g:[0,1] \times[0,+\infty) \rightarrow[0,+\infty), f:[0,1] \times[0,+\infty) \times[0,+\infty) \rightarrow[0,+\infty)$ and $k$ : $[0,+\infty) \rightarrow[0,+\infty)$ are continuous. It is easy to see that $g(t, x)$ is increasing in $x \in[0,+\infty)$ for fixed $t \in(0,1), k(y)$ is decreasing in $y \in[0,+\infty)$ and $f(t, x, y)$ is increasing in $x \in[0,+\infty)$ for fixed $t \in(0,1)$ and $y \in[0,+\infty)$, decreasing in $y \in[0,+\infty)$ for fixed $t \in(0,1)$ and $x \in$ $[0,+\infty)$. In addition, $\forall \eta \in(0,1)$ we get

$$
\begin{aligned}
& g(t, \eta x)=(\eta x(t))^{\frac{1}{4}}+t^{2} \geq \eta^{\frac{1}{4}}(x(t))^{\frac{1}{4}}+\eta t^{2} \geq \eta\left((x(t))^{\frac{1}{4}}+t^{2}\right) g(t, x)=\eta g(t, x), \\
& \begin{aligned}
f\left(t, \eta x, \eta^{-1} y\right) \geq \eta^{\frac{1}{4}}(x(t))^{\frac{1}{4}}+\eta^{\frac{1}{2}}(y(t)+1)^{-\frac{1}{2}}+1 \geq \eta^{\frac{1}{2}}\left((x(t))^{\frac{1}{4}}+\eta^{\frac{1}{2}}(y(t)+1)^{-\frac{1}{2}}+1\right) \\
=\eta^{\frac{1}{2}} f(t, x, y), \\
k\left(\eta^{-1} y\right)=\frac{1}{1+\eta^{-1} x} \geq \eta k(y) .
\end{aligned}
\end{aligned}
$$

Moreover, set $\delta_{1}=\delta_{2}=1$,

$$
\begin{aligned}
& f(t, x, y)=(x(t))^{\frac{1}{4}}+(y(t)+1)^{-\frac{1}{2}}+1 \geq \delta_{2} \geq \frac{1}{1+y}=k(y) \\
& f(t, x, y)=(x(t))^{\frac{1}{4}}+(y(t)+1)^{-\frac{1}{2}}+1 \geq(x(t))^{\frac{1}{4}}+t^{2}=\delta_{1} g(t, x) .
\end{aligned}
$$


Hence, all the conditions of Theorem 3.1 are satisfied. Then the problem (4.1) has a unique positive solution $x^{*}$ in $P_{h_{1}}$, where $h_{1}=t^{\frac{7}{2}}$.

\subsection{Example 2}

$$
\left\{\begin{array}{l}
D_{0^{+}}^{\frac{11}{3}} x(t)+t+t^{3}+\frac{2 x}{1+x}+\frac{1}{D_{0^{+}}^{\frac{5}{3}} x(t)+1}=0, \quad t \in(0,1) \\
x(0)=x^{\prime}(0)=x^{\prime \prime}(0)=0 \\
x^{\prime \prime}(1)=(x(1))^{-\frac{1}{3}}+5
\end{array}\right.
$$

Let $g(t, x)=t+\frac{x}{1+x}, f(t, x, y)=t^{3}+\frac{x}{1+x}+\frac{1}{y+1}$ and $k(y)=y^{-\frac{1}{3}}+5$. Obviously, $g:[0,1] \times$ $[0,+\infty) \rightarrow[0,+\infty), f:[0,1] \times[0,+\infty) \times[0,+\infty) \rightarrow[0,+\infty)$ and $k:[0,+\infty) \rightarrow[0,+\infty)$ are continuous. It is clear that $g(t, x)$ is increasing in $x \in[0,+\infty)$ for fixed $t \in(0,1), k(y)$ is decreasing in $y \in[0,+\infty)$ for fixed $t \in(0,1)$ and $f(t, x, y)$ is increasing in $x \in[0,+\infty)$ for fixed $t \in(0,1)$ and $y \in[0,+\infty)$, decreasing in $y \in[0,+\infty)$ for fixed $t \in(0,1)$ and $x \in[0,+\infty)$. In addition, $\forall \eta \in(0,1)$ we get

$$
\begin{aligned}
& g(t, \eta x)=t+\frac{\eta x}{1+\eta x} \geq \eta t+\eta \frac{x}{1+x}=\eta g(t, x) \\
& f\left(t, \eta x, \eta^{-1} y\right)=t^{3}+\frac{\eta x}{1+\eta x}+\frac{1}{\eta^{-1} y+1} \geq t^{3}+\frac{\eta x}{1+x}+\frac{\eta}{y+1} \geq \eta f(t, x, y), \\
& k\left(\eta^{-1} y\right)=\left(\eta^{-1} y\right)^{-\frac{1}{3}}+5 \geq \eta^{\frac{1}{3}} k(y) .
\end{aligned}
$$

Besides, $g(t, 0)=t \not \equiv 0$ and $f\left(t, 0, \frac{\Gamma(v)}{\Gamma(v-\beta)}\right)=t^{3}+\frac{1}{\frac{\Gamma(\nu)}{\Gamma(v-\beta)}+1} \not \equiv 0$, set $\delta_{0}=5$,

$$
f(t, x, y)+g(t, x)=t+t^{3}+\frac{2 x}{1+x}+\frac{1}{y(t)+1} \leq \delta_{0} \leq(y(t))^{-\frac{1}{3}}+5=k(y)
$$

Therefore, we proved all hypotheses of Theorem 3.2. Then we deduce that (4.1) has a unique positive solution $x^{*}$ in $P_{h_{2}}$, where $h_{2}=t^{\frac{8}{3}}$.

\subsection{Example 3}

Consider the following fractional differential equation:

$$
\left\{\begin{array}{l}
D_{0^{+}}^{\frac{9}{4}} x(t)+2+2(x(t))^{\frac{1}{4}}+\cos ^{2} t+\frac{1}{D_{0^{+}}^{\frac{5}{4}} x(t)+1}=0, \quad t \in(0,1), \\
x(0)=x^{\prime}(0)=0 \\
{\left[D_{0^{+}}^{\frac{3}{2}} x(t)\right]_{t=1}=\frac{1}{1+x(1)^{\frac{1}{2}}} .}
\end{array}\right.
$$

Let $g(t, x)=2+(x(t))^{\frac{1}{4}}, f(t, x, y)=\cos ^{2} t+(x(t))^{\frac{1}{4}}+\frac{1}{y+1}$ and $k(y)=\frac{1}{1+y^{\frac{1}{2}}}$. Evidently, $g:[0,1] \times$ $[0,+\infty) \rightarrow[0,+\infty), f:[0,1] \times[0,+\infty) \times[0,+\infty) \rightarrow[0,+\infty)$ and $k:[0,+\infty) \rightarrow[0,+\infty)$ are continuous. It is not difficult to verify that $g(t, x)$ is increasing in $x \in[0,+\infty)$ for fixed $t \in(0,1), k(y)$ is decreasing in $y \in[0,+\infty)$ and $f(t, x, y)$ is increasing in $x \in[0,+\infty)$ for fixed $t \in(0,1)$ and $y \in[0,+\infty)$, decreasing in $y \in[0,+\infty)$ for fixed $t \in(0,1)$ and $x \in[0,+\infty)$. Moreover, we get

$$
g(t, \eta x)=2+(\eta x(t))^{\frac{1}{4}} \geq \eta^{\frac{1}{4}} 2+\eta^{\frac{1}{4}}(x(t))^{\frac{1}{4}}=\eta^{\frac{1}{4}} g(t, x),
$$




$$
\begin{aligned}
& f\left(t, \eta x, \eta^{-1} y\right)=\cos ^{2} t+(x(t))^{\frac{1}{4}}+\frac{1}{\eta^{-1} y+1} \geq \eta \cos ^{2} t+\eta(x(t))^{\frac{1}{4}}+\frac{\eta}{y+1} \geq \eta f(t, x, y), \\
& k\left(\eta^{-1} y\right)=\frac{1}{1+\left(\eta^{-1} y\right)^{\frac{1}{2}}} \geq \frac{\eta^{\frac{1}{2}}}{1+y^{\frac{1}{2}}} \geq \frac{\eta}{1+y^{\frac{1}{2}}}=\eta k(y) .
\end{aligned}
$$

Besides, $f\left(t, 0, \frac{\Gamma(v)}{\Gamma(v-\beta)}\right)=\cos ^{2} t+\frac{1}{\frac{\Gamma(v)}{\Gamma(v-\beta)}+1} \not \equiv 0$, set $\delta_{1}=2, \delta_{2}=1$,

$$
\begin{aligned}
& k(y)=\frac{1}{1+y^{\frac{1}{2}}} \leq \delta_{1} \leq 2+(x(t))^{\frac{1}{4}}=g(t, x), \\
& f(t, x, y)=\cos ^{2} t+(x(t))^{\frac{1}{4}}+\frac{1}{y+1} \leq 2+(x(t))^{\frac{1}{4}}=\delta_{2} g(t, x) .
\end{aligned}
$$

In consequence, an application of Theorem 3.3 means that (4.1) has a unique positive solution $x^{*}$ in $P_{h_{3}}$, where $h_{3}=t^{\frac{5}{4}}$.

\section{Competing interests}

The authors declare that they have no competing interests.

\section{Authors' contributions}

LZ participated in the design of the study and drafted the main part of manuscript. HT carried out the theoretical studies and helped to draft the manuscript. All authors read and approved the final manuscript.

\section{Author details}

${ }^{1}$ College of Mathematics, Taiyuan University of Technology, 79 Yingze West Road, Taiyuan, Shanxi 030024, China. ${ }^{2}$ College of Mechanics, Taiyuan University of Technology, 79 Yingze West Road, Taiyuan, Shanxi 030024, China.

\section{Acknowledgements}

This paper is supported by opening project of State Key Laboratory of Explosion Science and Technology (Beijing Institute of Technology). The opening project number is KFJJ16-06M.

\section{Publisher's Note}

Springer Nature remains neutral with regard to jurisdictional claims in published maps and institutional affiliations.

Received: 19 September 2016 Accepted: 21 March 2017 Published online: 20 April 2017

\section{References}

1. Hilfer, R: Applications of Fractional Calculus in Physics. World Scientific, Singapore (2000)

2. Podlubny, I: Fractional Differential Equations. Mathematics in Science and Engineering. Academic Press, New York (1999)

3. Kilbas, AA, Srivastava, HM, Trujillo, Jj: Theory and Applications of Fractional Differential Equations. Elsevier, Amsterdam (2006)

4. Samko, SG, Kilbas, AA, Marichev, Ol: Fractional Integrals and Derivatives: Theory and Applications. Gordon and Breach, Yverdon (1993)

5. Baleanu, D, Machado, JAT, Luo, ACJ: Fractional Dynamics and Control. Springer, Berlin (2012)

6. Weitzner, H, Zaslavsky, GM: Some applications of fractional equations. Commun. Nonlinear Sci. Numer. Simul. 8(3-4), 273-281 (2003)

7. Zhai, CB, Yan, WP, Yang, C: A sum operator method for the existence and uniqueness of positive solutions to Riemann-Liouville fractional differential equation boundary value problems. Commun. Nonlinear Sci. Numer. Simul. $18,858-866(2013)$

8. Goodrich, CS: Existence of a positive solution to a class of fractional differential equations. Appl. Math. Lett. 23(9), 1050-1055 (2010)

9. Jeli, M, Samet, B: Existence of positive solutions to an arbitrary order fractional differential equation via a mixed monotone operator method. Nonlinear Anal., Model. Control 20(3), 367-376 (2015)

10. Yuan, CJ: Multiple positive solutions for $(n-1,1)$-type semipositone conjugate boundary value problems of nonlinear fractional differential equations. Electron. J. Qual. Theory Differ. Equ. 2010, 36 (2010)

11. Goodrich, CS: Existence of a positive solution to systems of differential equations of fractional order. Comput. Math. Appl. 62(3), 1251-1268 (2011)

12. Bai, Z, Lü, H: Positive solutions for boundary value problem of nonlinear fractional differential equation. J. Math. Anal. Appl. 311(2), 495-505 (2005)

13. Cheng, C, Feng, Z, Su, Y: Positive solutions for boundary value problem of fractional differential equation with derivative terms. Electron. J. Qual. Theory Differ. Equ. 2012, 215 (2012) 
14. Yang, W: Positive solutions for a coupled system of nonlinear fractional differential equations with integral boundary conditions. Comput. Math. Appl. 63(1), 288-297 (2012)

15. Ji, Y, Guo, YP, Qiu, JQ, Yang, LY: Existence of positive solutions for a boundary value problem of nonlinear fractional differential equations. Adv. Differ. Equ. 2015(1), 13 (2015)

16. Xu, XJ, Jiang, DQ, Yuan, CJ: Multiple positive solutions to singular positone and semipositone Dirichlet-type boundary value problems of nonlinear fractional differential equations. Nonlinear Anal. 74(16), 5685-5696 (2011)

17. Liu, LL, Zhang, XQ, Liu, LS, Wu, YH: Iterative positive solutions for singular nonlinear fractional differential equation with integral boundary conditions. Adv. Differ. Equ. 2016(1), 154 (2016)

18. Guo, LM, Liu, LS, Wu, YH: Existence of positive solutions for singular fractional differential equations with infinite-point boundary conditions. Nonlinear Anal., Model. Control 21(5), 635-650 (2016)

19. Guo, LM, Liu, LS, Wu, YH: Uniqueness of iterative positive solutions for the singular fractional differential equations with integral boundary conditions. Bound. Value Probl. 2016(1), 147 (2016)

20. Wang, $H$, Zhang, LL: The solution for a class of sum operator equation and its application to fractional differential equation boundary value problems. Bound. Value Probl. 2015(1), 203 (2015)

\section{Submit your manuscript to a SpringerOpen ${ }^{\mathcal{O}}$ journal and benefit from:}

- Convenient online submission

- Rigorous peer review

Immediate publication on acceptance

- Open access: articles freely available online

- High visibility within the field

- Retaining the copyright to your article 